\title{
Larson's third law and the universality of molecular cloud structure
}

\author{
M. Lombardi ${ }^{1,2}$, J. Alves ${ }^{3}$, and C. J. Lada ${ }^{4}$ \\ 1 University of Milan, Department of Physics, via Celoria 16, 20133 Milan, Italy \\ e-mail: mlombard@eso.org \\ 2 European Southern Observatory, Karl-Schwarzschild-Straße 2, 85748 Garching bei München, Germany \\ 3 University of Vienna, Türkenschanzstrasse 17, 1180 Vienna, Austria \\ 4 Harvard-Smithsonian Center for Astrophysics, Mail Stop 42, 60 Garden Street, Cambridge, MA 02138, USA
}

Received 25 June 2010 / Accepted 23 August 2010

ABSTRACT

\begin{abstract}
Larson (1981) first noted a scaling relation between masses and sizes in molecular clouds that implies that these objects have approximately constant column densities. This original claim, based upon millimeter observations of carbon monoxide lines, has been challenged by many theorists, arguing that the apparent constant column density observed is merely the result of the limited dynamic range of observations, and that in reality clouds have column density variations over two orders of magnitudes. In this letter we investigate a set of nearby molecular clouds with near-infrared excess methods, which guarantee very large dynamic ranges and robust column density measurements, to test the validity of Larson's third law. We verify that different clouds have almost identical average column densities above a given extinction threshold; this holds regardless of the extinction threshold, but the actual average surface mass density is a function of the specific threshold used. We show that a second version of Larson's third law, involving the mass-radius relation for single clouds and cores, does not hold in our sample, indicating that individual clouds are not objects that can be described by constant column density. Our results instead indicate that molecular clouds are characterized by a universal structure. Finally we point out that this universal structure can be linked to the log-normal nature of cloud column density distributions.
\end{abstract}

Key words. ISM: clouds - dust, extinction - ISM: structure - methods: statistical

\section{Introduction}

It has long been recognized that star formation is inextricably linked to the molecular clouds where the process is taking place, and therefore it is important to study the structure of these objects. One of the first attempts in this direction has been carried out by Larson (1981). In his seminal work, Larson used molecular line data available from earlier studies (mostly millimeter data of nearly objects) and showed that molecular clouds obey three scaling relations: (1) a power-law relationship between the length $L$ of the cloud and its velocity dispersion $\sigma_{\mathrm{v}}$, with $\sigma_{\mathrm{v}} \propto L^{0.38}$; (2) approximate virial equilibrium, with $2 G M / \sigma_{\mathrm{v}}^{2} L \simeq 1$; and (3) a relationship between the density $n$ of the cloud and its length, with $n \propto L^{-1.1}$. Larson's third law, which is the main focus of this letter, implies that molecular clouds have approximately constant column densities $\Sigma$, since $\Sigma \sim n L \propto L^{-0.1}$.

Since their formulation, Larson's laws have been the subject of several observational and theoretical studies. From the observational point of view, Solomon et al. (1987) presented ${ }^{12} \mathrm{CO}$ data for a 273 nearby clouds from the University of Massachusetts-Stony Brook (UMSB) Galactic Plane Survey (Sanders et al. 1986). They found a size-line width relationship with a power index $(0.5)$ steeper than the one derived by Larson (1981). Additionally, in agreement with Larson's third law, they found that the molecular gas surface density is approximately constant for all clouds with $\Sigma\left(\mathrm{H}_{2}\right)=170 M_{\odot} \mathrm{pc}^{-2}$. Recently, the same sample of clouds has been reanalysed by Heyer et al. (2009) using data from the Boston University-FCRAO Galactic Ring Survey (Jackson et al. 2006). The use of ${ }^{13} \mathrm{CO}(J=10)$ emission instead of ${ }^{12} \mathrm{CO}$ ensures that a large fraction of the data are optically thin; additionally, the data used have a much higher angular sampling and spectral resolution. Heyer et al. (2009) confirmed Larson's third law with a relative scatter (approximately a factor 3) similar to previous studies. However, surprisingly they found a median mass surface density of molecular hydrogen for this sample of $42 M_{\odot} \mathrm{pc}^{2}$, thus significantly smaller than the one derived by Solomon et al. (1987).

On the theoretical side, there have been many attempts to explain Larson's laws using numerical simulations. In many cases, the validity of Larsons relations, and especially of the third law, has been questioned (Kegel 1989; Scalo 1990; Vazquez-Semadeni et al. 1997; Ballesteros-Paredes \& Mac Low 2002; Ballesteros-Paredes 2006). In particular, it has been suggested that this law is merely the result of the limited dynamic range of observations, and that in reality mass surface densities of molecular clouds span at least two orders of magnitude.

In this letter, we re-examine the validity of Larson's third law using extinction as a tracer of molecular gas (Lada et al. 1994). The use of this tracer, in combination with advanced techniques (Lombardi \& Alves 2001; Lombardi 2009), allows us to probe clouds over a large dynamical range (typically more than two order of magnitudes in extinction); additionally, the column density measurements use a simple tracer, dust, which is not plagued by the uncertainties affecting millimeter observations of gas and dust (e.g., deviations from local thermodynamic equilibrium, opacity variations, chemical evolution, small-scale structure, depletion of molecules, unknown emissivity properties of the dust, unknown dust temperature).

The results of this study are twofold: first, we verify that Larson's law of constant column density holds with a very 
small scatter on a set of nearby clouds investigated using NICER (Lombardi \& Alves 2001) and NICEST (Lombardi 2009); second, we show that the same law, applied within a single cloud (using different extinction thresholds) as $M \propto L^{2}$ does not hold. Additionally, we argue that the first version of Larson's third law implies a universal physical structure for molecular clouds, which we identify in their log-normal distributions for the projected gas density.

Larson's third law, in its original formulation, links the average density $\left\langle n\left(\mathrm{H}_{2}\right)\right\rangle$ of clouds with their size $L:\left\langle n\left(\mathrm{H}_{2}\right)\right\rangle=$ $3400 \mathrm{~cm}^{-3}(L / 1 \mathrm{pc})^{\alpha}$, with $\alpha=-1.10$. Here $L$ is defined as the maximum observed linear extent of the cloud, and $\left\langle n\left(\mathrm{H}_{2}\right)\right\rangle$ is the average density of a sphere of diameter $L$ and total mass $M$ identical to the cloud (typically estimated from ${ }^{13} \mathrm{CO}$ data). Larson's data were more heterogeneous and included different clouds studied at different contours of integrated intensity, which resulted in a scatter of approximately one order of magnitude about the assumed relation; as we will see, our data suggest instead that Larson's law holds with a scatter below $15 \%$. The fact that $\alpha \simeq-1$ implies that the cloud projected column density, $\left\langle n\left(\mathrm{H}_{2}\right)\right\rangle L \propto L^{-0.1}$, is approximately constant. Larson discussed a few possible explanations for this: one-dimensional shock compressions, optical depth natural selection effects, and observational biases owing to the limited dynamic range of the ${ }^{13} \mathrm{CO}$ data.

\section{An extinction measurement of Larson's law}

\subsection{Definitions}

We consider first (Sect. 2.3) the following version of Larson's third law. Since we have at our disposal complete extinction maps, we can consider the area $S$ of a cloud above a given extinction threshold $A_{0}$ (throughout this letter, unless otherwise noted, we will refer to extinction measurements in the $K$ band, $A_{K}$, and drop everywhere the index $K$ ). We then define the cloud size implicitly from $S=\pi(L / 2)^{2}$ (or the cloud radius as $R=L / 2$ ). Similarly, we can consider the cloud mass $M$ above the same extinction threshold.

We will also briefly investigate the mass vs. radius relationship for each individual cloud, and verify whether we recover Larson's prediction $M(R) \propto R^{2}$ (Sect. 2.4). Note that the two versions of Larson's third law (different clouds above a fixed extinction threshold, or same cloud at various extinction thresholds) are clearly linked, but are not equivalent, in the sense that only one of the two might hold. Note also Larson (1981) defacto studied different clouds at different thresholds, and therefore used a mixture of both versions considered separately here.

Throughout this letter we will treat molecular complexes as single objects, and we will not split unconnected regions belonging to the same complex. Since typically a cloud will have many clumps with relatively high column densities, this procedure avoids the "creation" of new clouds when the extinction threshold $A_{0}$ is increased. This procedure is justified because our objects are mainly well defined regions, relatively far from the galactic plane, and with no or little contamination from other clouds.

\subsection{Data analysis}

The data used here are extinction maps obtained from the point source catalog of the Two Micron All Sky Survey (2MASS;

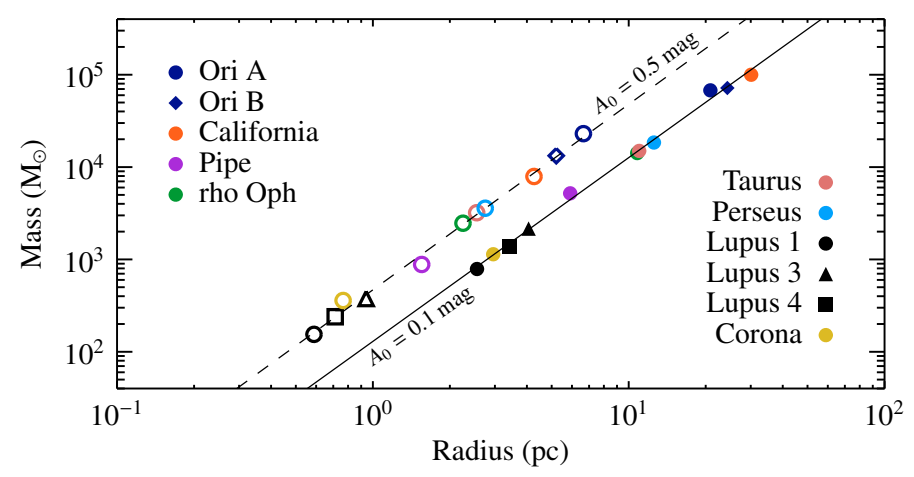

Fig. 1. Cloud masses above extinction thresholds of $A_{0}=0.1 \mathrm{mag}$ (filled symbols) and $A_{0}=0.5 \mathrm{mag}$ (open symbols) as a function of their size. The two line shows the best constant surface density fits, which correspond to $\Sigma=41 M_{\odot} \mathrm{pc}^{-2}$ and $\Sigma=149 M_{\odot} \mathrm{pc}^{-2}$ respectively.

Table 1. Best power-law fits $M=a \pi R^{\gamma}$ for various extinction thresholds.

\begin{tabular}{ccccc}
\hline \hline $\begin{array}{c}\text { Threshold } A_{0} \\
(\mathrm{mag})\end{array}$ & $\begin{array}{c}a \\
\left(M_{\odot} \mathrm{pc}^{-\gamma}\right)\end{array}$ & $\gamma$ & $\begin{array}{c}\text { Scatter } \\
(\text { percent })\end{array}$ & $c$ \\
\hline 0.1 & 41.2 & 1.99 & $11 \%$ & 2.25 \\
0.2 & 73.1 & 1.96 & $12 \%$ & 2.00 \\
0.5 & 149.0 & 2.01 & $14 \%$ & 1.63 \\
1.0 & 264.2 & 2.06 & $12 \%$ & 1.44 \\
1.5 & 379.8 & 2.07 & $14 \%$ & 1.38 \\
\hline
\end{tabular}

Notes. Note that because $\gamma \simeq 2$ in all cases, the quantity $a$ can be interpreted as the average mass column density of the cloud above the corresponding extinction threshold. The last two columns show the standard deviation of the cloud column densities divided by their average (relative scatter) and the ratio between the average column densities and the minimum column density set by the extinction threshold (c).

Kleinmann et al. 1994). Data for the various complexes have been reduced using NICER (Lombardi \& Alves 2001) and NICEST (Lombardi 2009) and following the prescriptions adopted in previous works (see Lombardi et al. 2006, 2008, 2010). The complexes considered are nearby molecular clouds, and therefore we are able to well resolve most cores with the 2MASS data; the same clouds have been used in Lada et al. (2010). Extinction measurements are converted into surface mass densities using

$\Sigma=\mu \mathrm{m}_{\mathrm{p}} \beta_{K} A_{K}$,

where $\mu$ is the mean molecular weight, $\beta_{K} \equiv[N(\mathrm{HI})+$ $\left.2 N\left(\mathrm{H}_{2}\right)\right] / A_{K} \simeq 1.67 \times 10^{22} \mathrm{~cm}^{-2} \mathrm{mag}^{-1}$ is the gas-to-dust ratio (Savage \& Mathis 1979; Lilley 1955; Bohlin et al. 1978), and $m_{\mathrm{p}}$ is the proton mass. With a standard gas composition $(63 \%$ hydrogen, $36 \%$ helium, and $1 \%$ dust) we have $\mu \simeq 1.37$ and therefore $\Sigma / A_{K} \simeq 183 M_{\odot} \mathrm{pc}^{-2} \mathrm{mag}^{-1}$.

\subsection{Larson's third law for a constant extinction threshold}

Figure 1 shows the amount of mass different clouds have above extinction thresholds of $A_{K}=0.1 \mathrm{mag}$ and $A_{K}=0.5 \mathrm{mag}$ as a function of the cloud "radii" (defined according to Sect. 2.1), together with the best power-law fit. As apparent from this plot, all clouds follow exquisitely well a Larson-type relationship, with $M \propto R^{2}$, and have therefore very similar projected mass densities at each extinction threshold. This result is also quantitatively shown in Table 1, where we report the best-fit power-laws for the mass vs. radius relation at different extinction thresholds. The 
M. Lombardi et al.: Larson's 3rd law and the universality of molecular cloud structure

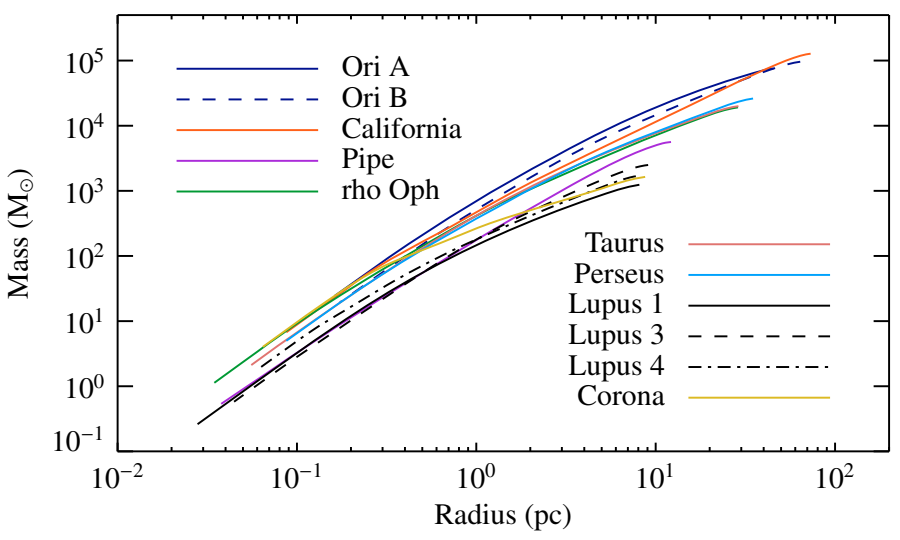

Fig. 2. Mass vs. radius relationship; both quantities are defined as indicated in Sect. 2.1.

exceptionally small scatter observed in Fig. 1 is also confirmed by the results shown in Table 1: at all extinctions considered, data follow the best-fit power-laws with relative standard deviations always below $15 \%$.

Table 1 also show the dimensionless factor $c$ obtained from the best quadratic fit $M=c \mu \mathrm{m}_{\mathrm{p}} \beta_{K} A_{0} \pi R^{2}$ in terms of the constants appearing in Eq. (1). Hence, $c=\left\langle A_{K}\right\rangle / A_{0} \geq 1$, and the fact that $c \sim 2$ with a very small relative scatter among different clouds indicates that all these objects have a very similar physical structure.

\subsection{Larson's third law for single clouds}

Figure 2 shows the second version of Larson's third law considered here, i.e. the mass vs. radius relationship. As apparent from this figure, the tracks for the various clouds have similar trends, but span a relatively large range of masses. In the range $R \in[0.1,1]$ pc we can fit a power-law of the form $M(R)=$ $380 M_{\odot}(R / \mathrm{pc})^{1.6}$, a result that compares well with the one obtained by Kauffmann et al. $(2010), M(R)=400 M_{\odot}(R / \mathrm{pc})^{1.7}$. Different clouds have quite similar exponents (the standard deviation of the power-law index is $\sim 0.18$ ), but rather different masses (the best-fit scale parameter for the mass ranges from 150 to $710 M_{\odot}$ ). Note, however, that since the power-law index is significantly different from two, errors on the assumed distances of the clouds would affect the scale parameter for the mass.

From this analysis we conclude that Larson's third law is not an accurate description of the mass vs. radius relationship for single clouds. Specifically, at larger scales all clouds show a flattening of the curves and deviates significantly from a powerlaw, while at smaller scales clouds follow power-laws, but with an exponent significantly different than two.

\subsection{Cloud physical structure}

As mentioned earlier, that an ensemble of clouds satisfies Larson's third law at different extinction thresholds suggests that clouds have a universal physical structure.

In order to investigate this point better, we consider in Fig. 3 the average column density of cloud material above a given extinction threshold, as a function of the extinction threshold. Figure 3 indicates a remarkable uniformity among the various clouds: they all show a relatively flat plateau up to $\sim 0.1 \mathrm{mag}$, and then a constant rise up to 2-5 mag. In the range $A_{0} \in$ $[0.1,1] \mathrm{mag}$, the curves for all clouds are confined within a relatively narrow region. In this extinction range we can fit a

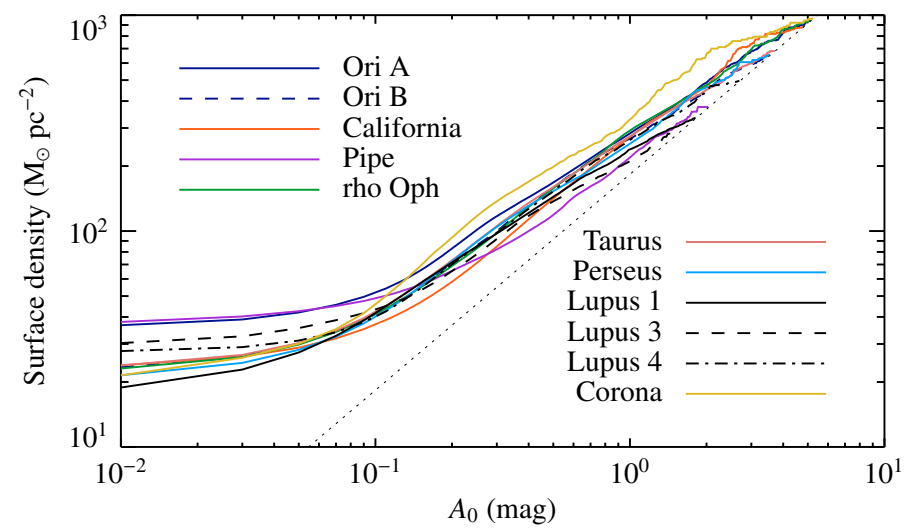

Fig. 3. Cloud mass surface density above an extinction threshold as a function of the threshold, in logarithmic scale. The dotted line shows the relationship between the cloud column density in $M_{\odot} \mathrm{pc}^{-2}$ and the extinction in the $K$ band.

simple power-law to the data plotted in Fig. 3, obtaining $\Sigma=$ $265 M_{\odot} \mathrm{pc}^{-2}\left(A_{0} / \mathrm{mag}\right)^{0.8}$. Note that an error analysis of the data points in Fig. 3 at $A_{0}<0.05$ mag shows that they are significant, because the large number of independent measurements contributing to these data make the statistical errors negligible, and because the flatness of the plateau at low extinction values makes them robust with respect to systematic errors (such as offsets in the NICER maps due to extinction in the control field).

\section{Theoretical interpretation}

The results presented above indicates that clouds have similar structures. Observationally (see, e.g., Lombardi et al. 2008; Kainulainen et al. 2009; Lombardi et al. 2010; Froebrich \& Rowles 2010), many clouds show a log-normal distribution at low extinctions:

$p_{A}(A)=\frac{1}{\sqrt{2 \pi} \sigma A} \exp \left[-\frac{\left(\ln A-\ln A_{1}\right)^{2}}{2 \sigma^{2}}\right]$,

where $A_{1}$ and $\sigma$ are two positive parameters. A tail at high extinctions, present in many clouds, is generally associated with the effects of gravitational instability. The log-normality of $p_{A}(A)$ is often linked with supersonic turbulence, although recent results show that this is also a common feature of very different classes of cloud models (Tassis et al. 2010).

Interestingly, we can express the mass and the area of a cloud above an extinction threshold as simple integrals of $p_{A}(A)$. Given a cloud of total area $S_{\text {tot }}$, the area and mass above a given extinction threshold $A_{0}$ are

$$
\begin{aligned}
& S\left(A_{0}\right)=S_{\text {tot }} \int_{A_{0}}^{\infty} p_{A}(A) \mathrm{d} A, \\
& M\left(A_{0}\right)=S_{\text {tot }} \mu \mathrm{m}_{\mathrm{p}} \beta \int_{A_{0}}^{\infty} A p_{A}(A) \mathrm{d} A .
\end{aligned}
$$

In particular, if we consider the log-normal distribution of Eq. (2), we obtain for the column density above $A_{0}$

$\Sigma\left(A_{0}\right) \equiv \frac{M\left(A_{0}\right)}{S\left(A_{0}\right)}=A_{1} \mu \mathrm{m}_{\mathrm{p}} \beta \kappa\left(A_{0} / A_{1}\right)$,

where $\kappa$ is a dimensionless quantity defined as

$\kappa(a)=\exp \left(\frac{\sigma^{2}}{2}\right) \frac{1-\operatorname{erf}\left[\left(\ln a-\sigma^{2}\right) / \sqrt{2} \sigma\right]}{1-\operatorname{erf}[\ln a / \sqrt{2} \sigma]}$. 


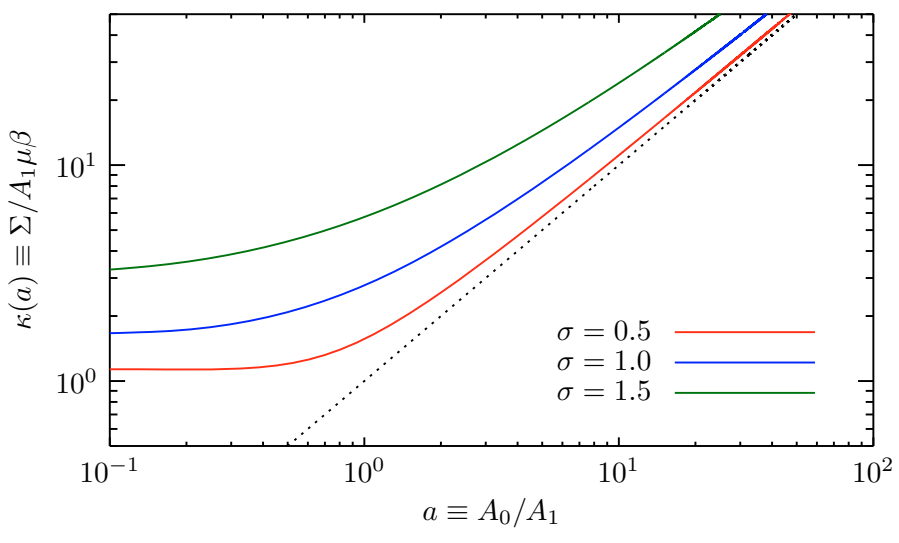

Fig. 4. Dimensionless column density $\kappa \equiv \Sigma / A_{1} \mu \mathrm{m}_{\mathrm{p}} \beta$, defined in Eq. (6), as a function of the dimensionless column density $a \equiv A_{0} / A_{1}$. The dotted line shows the asymptotic limit $\kappa(a) \simeq a$. Note the similarity with the curves shown in Fig. 3.

Table 2. Log-normal fit parameters for various clouds.

\begin{tabular}{lcc}
\hline \hline Cloud & $A_{1}$ & $\sigma$ \\
\hline Lupus V & 0.15 & 0.42 \\
Coalsack & 0.38 & 0.28 \\
Taurus & 0.18 & 0.49 \\
Lupus I & 0.08 & 0.43 \\
Ophiuchus & 0.16 & 0.48 \\
Serpens & 0.33 & 0.51 \\
Cha I & 0.11 & 0.35 \\
Cha II & 0.12 & 0.35 \\
Lupus III & 0.14 & 0.35 \\
Cor A & 0.10 & 0.44 \\
LDN 1228 & 0.12 & 0.32 \\
Pipe & 0.42 & 0.29 \\
\hline
\end{tabular}

\begin{tabular}{lcc}
\hline \hline Cloud & $A_{1}$ & $\sigma$ \\
\hline LDN 134 & 0.10 & 0.39 \\
LDN 204 & 0.14 & 0.41 \\
LDN 1333 & 0.12 & 0.38 \\
LDN 1719 & 0.15 & 0.50 \\
Musca & 0.08 & 0.45 \\
Cha 3 & 0.12 & 0.46 \\
Ori A GMC & 0.13 & 0.50 \\
Perseus & 0.13 & 0.48 \\
Ori B GMC & 0.11 & 0.49 \\
Cepheus & 0.16 & 0.59 \\
California & 0.14 & 0.51 \\
& &
\end{tabular}

Notes. Data are from Kainulainen et al. (2009) and converted into $K$-band extinction parameters using a standard reddening law (Rieke \& Lebofsky 1985).

We plot in Fig. 4 the function $\kappa(a)$ in a log-log scale for three values of the parameter $\sigma$. A comparison of Fig. 4 with Fig. 3 shows that the log-normal model is able to capture the main characteristics, except the "collapse" of curves onto the dotted line in Fig. 3 (most likely due to limited dynamic range of observations). Furthermore, in order to obtain the narrow bundle of curves in Fig. 3, the parameters $A_{1}$ and $\sigma$ for the various clouds must span a limited range. Typical relative scatters of $A_{1}$ and $\sigma$ are of the order of $57 \%$ and $19 \%$ (cf. Table 2). Additionally, an analytical calculation shows that the particular form of the log-normal distribution further suppresses these scatters (respectively by factors between 8 and 4), so that the final expected relative standard deviation in $\Sigma$ of the order of $14 \%$, in agreement with the data presented in Table 1.

\section{Summary}

1. Using near-infrared extinction maps of a set of nearby clouds we tested Larson's third law for molecular clouds, the constancy of average mass surface densities above a given extinction threshold. We verified this scaling law to a relatively high degree of precision. We found a very small $(<15 \%)$ relative scatter for the measured column densities independent of the adopted extinction thresholds over a very large range, from $A_{K}=0.1 \mathrm{mag}$ to $A_{K}=1.5 \mathrm{mag}$. Additionally, we found the value of the average mass surface density to be a function of the adopted extinction threshold.

2. We verified that Larson's third law does not hold when considering the mass-radius relation within single clouds. In the range $R \in[0.1,1]$ pc we find that the mass scales as $M(R) \propto R^{1.6}$, and is therefore significantly shallower than what was predicted by Larson; at larger radii, the relation appears to flatten even more.

3. We interpreted these results, and in particular item 1 above, as the effects of a universal physical structure shared among the different clouds. We showed that this universal structure is represented by a uniformity in the cloud density distributions. We found that a log-normal model is able to account for this uniformity, provided that the log-normal parameters are restricted to relatively narrow ranges. This suggests that Larson's third law might be a consequence of this special property of cloud structure.

Acknowledgements. We thank the referee for helping us to significantly improve this paper.

\section{References}

Ballesteros-Paredes, J. 2006, MNRAS, 372, 443

Ballesteros-Paredes, J., \& Mac Low, M. 2002, ApJ, 570, 734

Bohlin, R. C., Savage, B. D., \& Drake, J. F. 1978, ApJ, 224, 132

Froebrich, D., \& Rowles, J. 2010, MNRAS, 812

Heyer, M., Krawczyk, C., Duval, J., \& Jackson, J. M. 2009, ApJ, 699, 1092

Jackson, J. M., Rathborne, J. M., Shah, R. Y., et al. 2006, ApJS, 163, 145

Kainulainen, J., Beuther, H., Henning, T., \& Plume, R. 2009, A\&A, 508, L35

Kauffmann, J., Pillai, T., Shetty, R., Myers, P. C., \& Goodman, A. A. 2010, ApJ, 716,433

Kegel, W. H. 1989, A\&A, 225, 517

Kleinmann, S. G., Lysaght, M. G., Pughe, W. L., et al. 1994, Exper. Astron., 3, 65

Lada, C. J., Lada, E. A., Clemens, D. P., \& Bally, J. 1994, ApJ, 429, 694

Lada, C. J., Lombardi, M., \& Alves, J. F. 2010, ApJ, submitted

Larson, R. B. 1981, MNRAS, 194, 809

Lilley, A. E. 1955, ApJ, 121, 559

Lombardi, M. 2009, A\&A, 493, 735

Lombardi, M., \& Alves, J. 2001, A\&A, 377, 1023

Lombardi, M., Alves, J., \& Lada, C. J. 2006, A\&A, 454, 781

Lombardi, M., Lada, C. J., \& Alves, J. 2008, A\&A, 489, 143

Lombardi, M., Lada, C. J., \& Alves, J. 2010, A\&A, 512, A67

Rieke, G. H., \& Lebofsky, M. J. 1985, ApJ, 288, 618

Sanders, D. B., Clemens, D. P., Scoville, N. Z., \& Solomon, P. M. 1986, ApJS, 60,1

Savage, B. D., \& Mathis, J. S. 1979, ARA\&A, 17, 73

Scalo, J. 1990, in Physical Processes in Fragmentation and Star Formation, ed. R. Capuzzo-Dolcetta, C. Chiosi, \& A. di Fazio, AS\&S Library, 162, 151

Solomon, P. M., Rivolo, A. R., Barrett, J., \& Yahil, A. 1987, ApJ, 319, 730

Tassis, K., Christie, D. A., Urban, A., et al. 2010, MNRAS, in press [arXiv: 1006.2826$]$

Vazquez-Semadeni, E., Ballesteros-Paredes, J., \& Rodriguez, L. F. 1997, ApJ, 474,292 